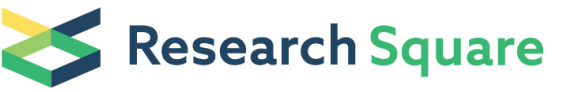 \\ Preprints are preliminary reports that have not undergone peer review. They should not be considered conclusive, used to inform clinical practice, or referenced by the media as validated information.
}

\section{Development and Application of Novel Indel Markers in Flax (Linum Usitatissimum L.) Through Whole-genome Re-sequencing}

\section{Hui Jiang}

Chinese Academy of Agricultural Sciences Institute of Bast Fiber Crops

\section{Gen Pan}

Chinese Academy of Agricultural Sciences Institute of Bast Fiber Crops

\section{Touming Liu}

Chinese Academy of Agricultural Sciences Institute of Bast Fiber Crops

\section{Li Chang}

Chinese Academy of Agricultural Sciences Institute of Bast Fiber Crops

\section{Siqi Huang}

Chinese Academy of Agricultural Sciences Institute of Bast Fiber Crops

\section{Huijuan Tang}

Chinese Academy of Agricultural Sciences Institute of Bast Fiber Crops

\section{Yuan Guo}

Chinese Academy of Agricultural Sciences Institute of Bast Fiber Crops

\section{Yena Wu}

Chinese Academy of Agricultural Sciences Institute of Bast Fiber Crops

\section{Jie Tao}

Chinese Academy of Agricultural Sciences Institute of Bast Fiber Crops

Anguo Chen ( $\square$ cagibfc@126.com)

Chinese Academy of Agricultural Sciences Institute of Bast Fiber Crops

\section{Research Article}

Keywords: Flax, InDel, Re-sequence, Oil content, Genetic diversity

Posted Date: August 23rd, 2021

DOI: https://doi.org/10.21203/rs.3.rs-700179/v2

License: (c) (i) This work is licensed under a Creative Commons Attribution 4.0 International License. Read Full License

Version of Record: A version of this preprint was published at Genetic Resources and Crop Evolution on January 18th, 2022. See the published version at https://doi.org/10.1007/s10722-021-01313-2. 


\section{Abstract}

Flax is an important oil and fibre crop grown in Northern Europe, Canada, India, and China. The development of molecular markers has accelerated the process of flax molecular breeding and has improved yield and quality. Presently, simple sequence repeat (SSR) and single nucleotide polymorphism (SNP) markers in the whole genome have been developed for flax. However, the development of flax insertion/deletion (InDel) markers has not been reported. A total of 17,110 InDel markers were identified by comparing whole-genome re-sequencing data of two accessions (87-3 and 84-3) with the flax reference genome. The length of InDels ranged from 1$277 \mathrm{bp}$, with 1-15 bp accounting for the highest rate (95.55\%). The most common InDels were in the form of single nucleotide (8840), dinucleotide (3700), and trinucleotide (1349), and chromosome 2 (1505) showed the highest number of InDels among flax chromosomes, while chromosome 10 (913) presented with the lowest number. From 17,110 InDel markers, 90 primers that were evenly distributed in the flax genome were selected. Thirty-two pairs of polymorphic primers were detected in two flax accessions, and the polymorphism rate was $40.70 \%$. Furthermore, genetic diversity analysis, population structure and principal component analyse (PCA) divided 69 flax accessions into two categories, namely oilseed flax and fibre flax using 32 pairs of polymorphic primers. Additionally, correlation analysis showed that InDel-26 and InDel-81 were associated with oil content traits, and two candidate genes (Ius10031535 and lus10025284) tightly linked to InDel-26 or InDel-81, might be involved in flax lipid biosynthesis and lipid metabolism. This study is the first to develop InDel markers based on re-sequencing in flax and clustered the markers into two well-separated groups for oil and fibre. The results demonstrated that InDel markers developed herein could be used for flax germplasm identification, genetic diversity analysis, and molecular marker-assisted breeding.

\section{Introduction}

Flax (Linum usitatissimum L., $2 \mathrm{n}=30$ ) is an important economic crop that is mainly cultivated in Canada, China, America, and India. Flax is a multipurpose plant with a high comprehensive utilisation value and is used in food, medicine, and feed, while fibre is widely used in manufacturing industries and in the development of composite materials and fireproof materials (Westcott and Muir 2003; Zuk et al. 2015). Therefore, cultivation of high-yield and high-quality flax varieties has emerged as one of the current breeding goals. Molecular markerassisted breeding (MAS) is a new type of breeding technology that can help achieve the breeding goal of increasing crop yield and quality in a more direct, rapid, and efficient manner. In recent years, with the development of sequencing technology, development of high-quality molecular markers in the whole genome has been realised. Random amplified polymorphic DNA (RAPD) (Fu et al. 2002; Fu et al. 2003; Kumari et al. 2018), amplified fragment length polymorphism (AFLP) (Spielmeyer et al. 1998; Chandrawati et al. 2014), simple sequence repeats (SSR) (Cloutier et al. 2009; Cloutier et al. 2011; Soto-Cerda et al. 2011; Asgarinia et al. 2013; Chandrawati and Yadav 2017; Wu et al. 2017; Pan et al. 2020), and single nucleotide polymorphism (SNP) (Kumar et al. 2012; Yi et al. 2017; Xie et al. 2018; Soto-Cerda et al. 2018; You et al. 2018; Singh et al. 2019; Hoque et al. 2020) molecular markers have been identified in flax. However, there no research has been conducted on flax InDel molecular markers. Therefore, the development of InDel for the expansion of molecular markers can accelerate the process of flax molecular breeding, thereby aiding the cultivation of high-quality flax varieties. 
AFLP, RAPD, SSR, and SNP markers in flax have been studied and used in germplasm identification, genetic diversity analysis, genetic map construction, QTL mapping, and genome-wide association analysis. Presently, SNPs are mainly used for genetic map construction (Yi et al. 2017; Zhang et al. 2018), QTL mapping (Wu et al. 2018; Zhang et al. 2018), and genome-wide association analysis (You et al. 2018; Xie et al. 2018; Soto-Cerda et al. 2018; Xie et al. 2019). Based on principles of the SLAF technology, a genetic map with the highest density using the best quality of flax was constructed (Yi et al. 2017; Wu et al. 2018), and the QTL loci and candidate genes related to the agronomic traits of flax were screened through association analysis (Xie et al. 2018). The SSR markers were widely used to analyse the genetic diversity of flax, and flax was divided into oil, fibre, and oilfibre flax according to its use (Wu et al. 2017; Pan et al. 2020). However, few studies have shown that the classification of flax varieties differs depending on the type of marker used (Wu et al. 2017). Although studies have been conducted on different types of molecular markers in flax, the continuous development of new molecular markers can help provide technical support for the study of molecular marker-assisted breeding of flax. With the development of re-sequencing technology in recent years, mining of high-quality molecular markers based on the whole genome can accelerate the research on flax molecular breeding.

With the development of high-throughput sequencing technology, the development of SNP and InDel markers based on the whole genome has been widely considered in crop molecular marker-assisted breeding (MAS). InDel refers to the insertion or deletion of nucleotides in the plant genome, and such nucleotides are widely distributed, dense, and abundant in the plant genome (Lv et al. 2013; Liu et al. 2013; Liu et al. 2016). InDel characteristics are similar to those of SSR, and InDel markers are composed of short fragments of different lengths. Owing to the low cost and high efficiency of sequencing, InDel can be developed easily using highthroughput sequencing (Liu et al. 2016; Guo et al. 2019). InDel markers have been used in rice (Hayashi et al. 2006; Wu et al. 2013; Sahu et al. 2017), jute (Yang et al. 2018), quinoa (Zhang et al. 2017), cabbage (Lv et al. 2013), cucumber (Adedze et al. 2021), tomato, (Ngan et al. 2016) and other crops (Liu et al. 2013; Liu et al. 2019; Guo et al. 2019; Kizil et al. 2020; Wang et al. 2020), and are mainly used for germplasm resource identification, genetic diversity analysis, and genetic map construction. However, there are no reports available on the development and application of InDel markers in flax.

To accelerate the application of more molecular markers in flax breeding, for the first time, re-sequencing was used to identify and develop flax InDel markers. In this study, based on the two materials with different oil contents developed in our laboratory in the early stage, our aims were to: 1 ) re-sequence the two materials (87 3 and $84-3)$ and compare them with the reference genome sequence to develop the InDel marker; 2) design the InDel primers and evaluate the quality of InDel markers use the two accessions ( 87 - 3 and $84-3$ ); 3 ) use polymorphic primers to analyse the genetic diversity of 69 flax accessions; and 4) conduct correlation analysis between InDel markers and oil content traits, as well as perform the screening of candidate genes related to oil content traits.

\section{Methods}

Plant materials

In the early stage, we prepared $87-3$ (male, $35.94 \%$ ) and $84-3$ (female, $45.77 \%$ ) samples with different oil contents for re-sequencing, and a total of 69 flax accessions were collected for genetic analysis. Among them, 10 accessions were provided by the Gansu Academy of Agricultural Sciences, 20 accessions were provided by 
the Heilongjiang Academy of Agricultural Sciences, and 37 private accessions were obtained (Table S1). These flax accessions were derived from regions across the globe and were divided into oil and fibre according to their use. All materials were planted in the western centre of the Chinese Academy of Agricultural Sciences, and the oil content of flaxseed was determined by conducting near-infrared spectral analysis (NIRS) after maturity was reached.

DNA sample preparation and re-sequencing

Young leaf tissues of 10 individuals of $84-3$ and $87-3$ were collected for performing de novo genomic resequencing. Fresh and young leaf tissues of 69 flax accessions at the first branching stage were collected, and DNA extraction was performed using the CTAB method (Murray and Thompson 1980). DNA quality and quantity were determined using an Eppendorf BioSpectrometer (Eppendorf, Hamburg, Germany). DNA quantification was further conducted using a fluorometer and DNA samples were diluted to a $10-\mathrm{ng} / \mathrm{L}$ working solution.

InDel detection and primer design

The flax reference genome sequence was obtained from NCBI

(https://www.ncbi.nlm.nih.gov/assembly/GCA_000224295.2) with the re-sequencing data of $87-3$ and $84-3$ to detect the InDel loci. The Primer 3.0 software (http://pgrc.ipk-gatersleben.de/misa/primer3.html) was used to design primers for the InDel loci. One pair of primers with the highest scoring was selected from the experimental design results.

InDel genotyping

Primers were designed based on the detected InDel loci. According to the distance of every $4 \mathrm{Mb}$ of the whole genome, InDel primers evenly distributed on the flax chromosome were selected for polymorphism analysis. InDel-primed polymerase chain reactions (PCRs) were performed in $10 \mu \mathrm{L}$ reaction volumes, which included 8 $\mu \mathrm{L}$ of the PCR mix solution (CWBIO, Beijing, China), $0.5 \mu \mathrm{L}$ of the forward primer $(10 \mathrm{nmol} / \mathrm{L}), 0.5 \mu \mathrm{L}$ of the reverse primer $(10 \mathrm{nmol} / \mathrm{L})$, and $1 \mu \mathrm{L}$ of the DNA template. PCR amplification was performed under the following conditions: (1) pre-denaturation at $95^{\circ} \mathrm{C}$ for $3 \mathrm{~min}$; (2) 35 cycles of $94^{\circ} \mathrm{C}$ for $30 \mathrm{~s}, 55^{\circ} \mathrm{C}$ for $30 \mathrm{~s}$, and $72^{\circ} \mathrm{C}$ for $1 \mathrm{~min}$; and (3) $72^{\circ} \mathrm{C}$ for $5 \mathrm{~min}$.

Genetic diversity assay and population structure analyse

The two materials ( 87 - 3 and $84-3$ ) were used for InDel primer polymorphism detected, and band types were detected by performing analysis using polypropylene gel. The genetic diversity of 69 flax accessions was analysed with polymorphic primer. Each polymorphic band detected using the same primer represented an allelic mutation. To generate molecular data matrices, clear bands for each fragment were scored for each accession for each primer pair and were recorded as 1 (presence of a fragment), 0 (absence of a fragment), and 9 (complete absence of band). The PowerMarker software (version 3.25) was used to provide data on basic summary statistics (Liu and Muse 2005), which included the polymorphism information content (PIC), the number of alleles (Na), major allele frequency (MAF), and gene diversity for each InDel. A clustering map was constructed based on the genetic distances and the unweighted pair-group method with arithmetic means (UPGMA) using MEGA V6.0. 
The population structure of 69 flax accessions was estimated using the software STRUCTURE v2.3.4. The number of presumed populations $(K)$ was set from 1 to 10 and running 10 times per round, burn in time and MCMC (Markov Chain Monte Carlo) replication number both to were set to 100,000. The STRUCTURE HARVESTER (Earl et al. 2012) was used to calculate the optimal $\mathrm{K}$ value to determine the most suitable number of sub-populations. In order to further analyse genetic relationships of flax accessions, a principal component analyse (PCA) was performed based on variability trends of InDel using the NTSYSpc version 2.10.

Correlation analysis between InDel markers and oil content traits

Correlation analysis was conducted using the SPSS software (version 13.0) to analyse the correlation between the InDel markers that could clearly be used to distinguish 69 flax accessions and the oil content trait data. Pearson correlation analysis was used to analyse the correlation coefficient between the flax oil content traits and InDel markers. A multiple linear stepwise regression method (Kraakman et al. 2004) was used to fit the InDel markers that showed significant correlation with the oil content traits of flax. The X-axis indicates the genotype generated with the presence of each InDel marker, and the Y-axis indicates the trait of flax oil content.

\section{Results}

Distribution of InDel markers

The clean base quantity generated was $3.69 \mathrm{G}$ for $84-3$ and was $1.01 \mathrm{G}$ for $87-3$ with an average of $2.35 \mathrm{G}$. Using the Burrows-Wheeler Alignment (BWA), $3.55 \mathrm{G}$ and $0.96 \mathrm{G}$ of $84-3$ and $87-3$ were respectively mapped to the flax reference genome sequence. The mapping read depth was $18.93 \times$ for $84-3$ and $6.02 \times$ for $87-3$, respectively (Table 1). Genome-wide InDel polymorphism led to the generation of 17,110 InDels between $84-3$ and 87 - 3 (Table S2), exhibiting an InDels density of $54.12 \mathrm{InDel} / \mathrm{Mb}$. InDel sites varied from 1-277 bp, and the number of InDel sites decreased as the length of InDel increased (Fig. 1). The number of InDels of 1-3 bp showed the highest value (81.17\%), followed by that of InDels of 4-15 bp (14.37\%); the number of InDels of > $15 \mathrm{bp}$ showed the least value (4.45\%). Among all InDels, the most common InDel sites were single nucleotide (8840) accounting for $51.67 \%$, dinucleotide (3700) accounting for $21.62 \%$, and trinucleotide (1349) accounting for $7.88 \%$. Furthermore, the repeats of A (2524) and T (2551) were more commonly present in single nucleotide sites, and there were four repeat types of dinucleotide (CA, CT, TA, and AT) and trinucleotide (TTC, AAT, CTT, and TAA) (Fig. S1). Additionally, the distribution of InDels on each chromosome was also different (Fig. 2A). Chromosome 2 exhibited the highest number of InDels (1505), and chromosome 10 demonstrated the lowest number (913). The average density of InDels in the whole genome of flax was $54.12 \mathrm{InDels} / \mathrm{Mb}$, and the InDel density of each chromosome was different. The highest density of chromosome 5 was $64.97 \mathrm{InDels} / \mathrm{Mb}$, while the lowest density of chromosome 8 was 40.35 InDels/Mb (Fig. 2B).

InDel marker polymorphism analysis

Based on data comprising 17,110 InDels, 17,110 primer pairs were designed. Ninety pairs of InDel primers were selected according to the distance of each $4 \mathrm{MB}$ and a difference of more than three bases in the whole genome, as shown in Table S3. The length of all primers was $24 \mathrm{bp}$, and the size of the product was 80-250bp. The effectiveness of 90 pairs of InDel primers showed that 86 primers led to the successful amplification of bands, 32 pairs of polymorphic primers, and the polymorphism rate was $40.70 \%$ (Fig. 3; Fig. S2). 
Data on 32 InDel primers were used to analyse the genetic diversity of the 69 flax accessions (Fig. 4). The number of alleles ( $\mathrm{Na}$ ) ranged from 2-3 (average of 2.25) (Table S4). Twenty-four InDels exhibited two alleles, and eight InDels presented with three alleles. The polymorphism information content (PIC) varied from 0.06740.3958 , with an average of 0.3097 , and the gene diversity ranged from $0.0698-0.5138$, with an average of 0.3899. Additionally, the average major allele frequency (MAF) was 0.6941, which varied from 0.5000-0.9638. InDel-51 presented with the highest PIC (0.3958) and gene diversity (0.5138). InDel-13 presented with the lowest PIC (0.0674), gene diversity (0.0698), and the highest MAF (0.9638).

The genetic relationships between the 69 flax accessions were analysed. As shown in Fig. 5, the 69 accessions were unambiguously classified into two clusters according to oil and fibre content. Group I included 35 accessions of oil. Group II included 34 accessions of fibre. However, the two accessions for oil (Baya 7, Kuerle) were classified into Group II. Moreover, five InDel markers (InDel-14, InDel-26, InDel-40, InDel-74, and InDel-81) could be used to largely classify 69 flax accessions for oil and fibre among the 32 polymorphic primers, and the five markers could be further analysed (Fig. 4; Fig. S3).

Population structure and principal component analyse

In order to further determine the population structure of 69 flax germplasm, STRCUTURE 2.3.4 was used to analyse the population structure. The results of Delta $K$ values of different assumed $(K)$ populations show that a sharp peak was given at $\mathrm{K}=2$, which indicated that there were two sub-populations in 69 flax accessions (Fig. 6A). As shown in Fig. 6B, the population structure analyse classified 69 flax genotypes into two subpopulations, with 33 fibre genotypes in population 1 and 36 oil genotypes in population 2. In addition, PCA analyse divided all accessions into two major groups (Fig. 7). The results of the two-dimensional scatter plot showed that principal component 1 and principal component 2 account for $63.7 \%$ and $21.0 \%$ of the variation, respectively.

Correlation analyse and candidate gene prediction

To further explore InDel markers related to oil content traits of flax, correlation analysis between five InDel markers and oil content traits was conducted (Table 2). The results showed that InDel-26 exhibited a significant positive correlation with oil content traits, whereas InDel-81 exhibited a significant negative correlation $(p<$ 0.05). Meanwhile, the correlation analysis between InDel markers and flax type InDel-26 showed the existence of an extremely significant negative correlation, while InDel-81 showed the existence of an extremely significant positive correlation $(p<0.01)$ (Table 3$)$.

Further, the marker loci information was compared with the flax reference genome data to identify the gene locus related to the two markers. Indel-26 and Indel-81 were located on chromosomes 12 and 8, respectively. The genes close to InDel-26 were lus10031534 and lus100315535, and functional annotations were found to be RNA-binding (RRM/RBD/RNP motifs) family protein and serine/threonine protein phosphatase $2 \mathrm{~A} 55 \mathrm{kDa}$ regulatory subunit B prime gamma, respectively (Table 4). The genes close to InDel-81 were lus10025284 and lus10010835, and their functional annotations included Lipin family protein and protein kinase superfamily protein, respectively. 
Table 1 Summary of the original sequencing data of $84-3$ and $87-3$

\begin{tabular}{lllll} 
Samples & Clean base (G) & Map ratio (\%) & Q20 (\%) & Depth \\
\hline $84-3$ & 3.69 & 96.34 & 98.11 & 18.93 \\
\hline $87-3$ & 1.01 & 95.1 & 98.01 & 6.02 \\
\hline Average & 2.35 & 95.72 & 98.06 & 12.475
\end{tabular}

Table 2 Correlation coefficients between InDel markers and oil content traits in flax

\begin{tabular}{|llllll|}
\hline Marker & Oil content & InDel-14 & InDel-26 & InDel-40 & InDel-74 \\
\hline InDel-14 & -0.015 & & & & \\
\hline InDel-26 & $0.278^{\star}$ & 0.011 & & & \\
\hline InDel-40 & -0.186 & 0.023 & 0.106 & & \\
\hline InDel-74 & -0.070 & $0.743^{\star \star}$ & 0.145 & 0.123 & \\
\hline InDel-81 & $-0.543^{\star \star}$ & 0.060 & -0.118 & $0.480^{\star \star}$ & 0.228 \\
\hline
\end{tabular}

* $P<0.05$ indicates a significant correlation.

** $P<0.01$ indicates an extremely significant correlation.

Table 3 Correlation coefficients between InDel markers and type of flax

\begin{tabular}{|clllll|}
\hline Marker & Type & InDel-14 & InDel-26 & InDel-40 & InDel-74 \\
\hline InDel-14 & 0.083 & & & & \\
\hline InDel-26 & $-0.475^{\star \star}$ & 0.011 & & & \\
\hline InDel-40 & $0.250^{\star}$ & 0.023 & 0.106 & & \\
\hline InDel-74 & 0.175 & $0.743^{\star \star}$ & 0.145 & 0.123 & \\
\hline InDel-81 & $0.639 * \star$ & 0.060 & -0.118 & $0.480^{\star \star}$ & 0.228 \\
\hline
\end{tabular}

$* P<0.05$ indicates a significant correlation.

** $P<0.01$ indicates an extremely significant correlation.

Table 4 Candidate gene information of InDel markers related to oil content traits in flax 


\begin{tabular}{|c|c|c|c|c|c|c|c|}
\hline Marker & Pos & Chr & ID & scaffold & Start & End & $\begin{array}{l}\text { Functional } \\
\text { annotation }\end{array}$ \\
\hline \multirow[t]{2}{*}{$\begin{array}{l}\text { InDel- } \\
26\end{array}$} & 20001016 & 12 & Lus10031534 & scaffold863 & 20003169 & 20003873 & $\begin{array}{l}\text { RNA-binding } \\
\text { (RRM/RBD/RNP } \\
\text { motifs) family } \\
\text { protein }\end{array}$ \\
\hline & & & Lus10031535 & scaffold863 & 19995796 & 19998319 & $\begin{array}{l}\text { Serine/threonine } \\
\text { protein } \\
\text { phosphatase } 2 \mathrm{~A} \\
55 \mathrm{kDa} \\
\text { regulatory } \\
\text { subunit B prime } \\
\text { gamma }\end{array}$ \\
\hline \multirow[t]{2}{*}{$\begin{array}{l}\text { InDel- } \\
81\end{array}$} & 12546472 & 8 & Lus10025284 & scaffold339 & 12554843 & 12558982 & $\begin{array}{l}\text { Lipin family } \\
\text { protein }\end{array}$ \\
\hline & & & Lus10010835 & scaffold440 & 12531041 & 12533379 & $\begin{array}{l}\text { Protein kinase } \\
\text { superfamily } \\
\text { protein }\end{array}$ \\
\hline
\end{tabular}

\section{Discussion}

In this study, re-sequencing was performed for the first time to develop InDels related to the oil content of flax. Based on the re-sequencing data, $17,110 \mathrm{InDels}$ were detected with a density of $54.12 \mathrm{InDel} / \mathrm{Mb}$ in the whole genome. Compared with jute (Corchorus spp.) (51,172) (Yang et al. 2018), quinoa (Chenopodium quinoa Willd.) $(842,783)$ (Zhang et al. 2017), apple (Malus ssp.) $(25,924)$ (Wang et al. 2020), tea plant (Camellia sinensis (L.) O. Kuntze) $(255,218$ ) (Liu et al. 2019), and pepper (Capsicum spp. L.) $(1,651,856)$ (Guo et al. 2019), the number of InDels detected in this study was low but was higher than that of cucumber (Cucumis sativus) $(10,470)$ (Adedze et al. 2021) and sesame (Sesamum indicum L.) (7,477) (Anonymous 2020). Compared with other markers in flax, the number of InDels was less than that of SNPs (Yi et al. 2017; You et al. 2018) and more than most SSR markers (Cloutier et al. 2009; Soto-Cerda et al. 2011; Cloutier et al. 2012; Wu et al. 2017). The main reason for obtaining such results may be attributed to the fact that compared with the complicated operations of RAPD, AFLP, and SSR, the use of a re-sequencing technology can aid an easy detection and obtainment of a larger number and higher quality of SNPs and InDels in the whole genome. Moreover, the length of InDel obtained in this study was 1-277 bp, with the largest number of single nucleotides (51.67\%), followed by dinucleotides (21.62\%) and trinucleotides (7.88\%). The small InDel fragments (1-15bp) accounted for a high rate $(95.55 \%)$. Additionally, A and T were the most commonly occurring single nucleotides, and these results were consistent with reported by previous studies (Yang et al. 2018; Liu et al. 2013; Wei et al. 2014). The InDel marker refers to the difference in nucleotides in the plant genome. In this study, using $84-3$ and $87-3$ with different oil contents constructed in the early stage of experiments, the InDel marker was developed for the first time in flax by re-sequencing, which was helpful for conducting flax molecular marker-assisted breeding.

Analysis of the genetic relationship between species has important statistical significance for guiding crop genetic breeding. To verify the developed InDel markers, 32 pairs of polymorphic primers were screened from 90 pairs of InDel markers evenly distributed on the chromosomes in 37 flax accessions. The polymorphism rate 
was $40.70 \%$, which was lower than that of pepper (67.4\%) (Guo et al. 2019), cucumber (54.81\%) (Adedze et al. 2021), and Brassica rapa (77\%) (Liu et al. 2013). Additionally, the PIC obtained was low (0.3097), which was consistent with the results reported by previous studies (Soto-Cerda et al. 2011; Wu et al. 2017; Pan et al. 2020). This indicated that InDel was more suitable for the identification of flax germplasm resources, since the degree of variation of flax genes was not substantial and the genetic background was narrow.

Genetic diversity analysis can help identify germplasm resources that play a key role in crop breeding through the genetic evolution of crops. Based on the results of the previous analysis, 32 polymorphic InDel markers could be used to divide 69 flax accessions into two groups, namely oil and fibre. Group I was established for oil, and Group II was established for fibre. This classification was consistent with that reported in previous studies (Chandrawati et al. 2014; Wu et al. 2017; Pan et al. 2020). In addition, population structure analyse showed that when Delta $\mathrm{K}$ had a peak at $\mathrm{K}=2,69$ flax genotypes were divided into two sub-populations, indicating that 69 flax accessions has a relatively simple population structure and a single genetic source. The PCA results also divided all accessions into two categories. The population structure and PCA classification results were consistent with the UPGMA clustering results, indicated that the classification results obtained in this study were reliable.

In previous studies, high-throughput sequencing techniques have been used for the development of SSR and SNP markers for QTL mapping and GWAS analysis of flax-related quality traits (Chandrawati and Yadav 2017; You et al. 2018; Xie et al. 2018; Xie et al. 2019). In this study, two InDel markers (InDel-26 and InDel-81) related to flax oil content traits were identified through correlation analysis of the developed InDel markers. Traditional breeding is the major method for the development of flax varieties, and molecular breeding remains in the exploratory stage. In this study, the InDel marker (InDel-26, InDel-81) could be used for the selection of traits with high oil content during flax breeding, and might further accelerate the process of flax molecular breeding.

Furthermore, through the comparison of gene loci information, four gene loci (Ius10031534, Ius10031535, Ius10025284, and Ius10010835) near InDel-26 and InDel-81 were found. Ius10031535 is functionally annotated as serine/threonine protein phosphatase $2 \mathrm{~A} 55 \mathrm{kD}$ a regulatory subunit $\mathrm{B}$ prime gamma, and the homologous gene in Arabidopsis thaliana is PUX5 (UniProtKB: Q7Y175; GenelD: 827210), which encodes a protein containing the ubiquitin regulatory domain. In Pichia pastoris, Ubx 1 and Ubx2 were reported to play a key role in the synthesis of unsaturated fatty acids by exerting influence on Spt23, and both are reportedly involved in the formation of lipid droplets and protein degradation (Zhang et al. 2017). lus10025284 is functionally annotated as a lipid family protein, and its homologous gene in Arabidopsis thaliana is PAH1 (UniProtKB: Q9SF47; GenelD: 820113), which catalyses the dephosphorylation of phosphates to generate diacylglycerols (Nakamura et al. 2009; Eastmond et al. 2010; Mietkiewska et al. 2011). Therefore, it is speculated that /us10031535 and Ius10025284 may function in flax lipid biosynthesis and lipid metabolism; however, the function of these genes in flaxseed warrants further verification using transgenic experiments.

\section{Conclusion}

In this study, 17,110 InDel markers were developed based on re-sequencing, and thirty-two pairs of polymorphic primers were obtained among accessions 84-3 and 87-3. Thereafter, 69 accessions were divided into two groups (oil and fibre) using 32 polymorphic primers based on population structure analysis, PCA analyisis and the UPGMA clustering. Furthermore, the correlation analysis showed that InDel-26 and InDel-81 were related to 
the oil content of flax, and two candidate genes (Ius10031535 and Ius10025284) may relate to lipid biosynthesis and lipid metabolism. This is the first study conducted on the development of InDel markers based on re-sequencing in flax, which may provide a theoretical basis for the identification of flax germplasm resources, genetic map construction, QTL mapping, and GWAS.

\section{Declarations}

Author contribution Anguo Chen designed experiment and provided resources. Hui Jiang performed experiment, analysed data and wrote original draft. Gen Pan designed experiment, analysed data and edited article.

Touming Liu designed experiment. Li Chang and Siqi Huang edited artical. Huijuan Tang investigated data of traits. Yuan Guo provide resources. Yena Wu and Jie Tao performed part of experiment.

Founding This research was funded by National Natural Science Foundation of China (31771852 and 31771853) and Special college-level overall planning project for basic scientific research business expenses of Chinese Academy of Agricultural Sciences (Y2019XK15-06).

Data availability All data generated or analyzed during this study are included in this published article and its supplementary information files.

Ethics approval and consent to participate Not applicable.

Consent for publication Not applicable.

Conflict of interest The authors declare no competing interests.

\section{References}

Adedze YMN, Lu X, Xia Y, Sun Q, Nchongboh CG, Alam MA, Liu M, Yang X, Zhang W, Deng Z, Li W, Si L (2021) Agarose-resolvable InDel markers based on whole genome re-sequencing in cucumber. Sci Rep 11:3872. https://doi.org/10.1038/s41598-021-83313-x

Asgarinia P, Cloutier S, Duguid S, Rashid K, Mirlohi AF, Banik M, Saeidi G (2013) Mapping quantitative trait loci for powdery mildew resistance in flax (Linum usitatissimum L.). Crop Sci 53: 2462-2472. https://doi.org/10.2135/cropsci2013.05.0298

Chandrawati, Maurya R, Singh PK, Ranade SA, Yadav HK (2014) Diversity analysis in Indian genotypes of linseed (Linum usitatissimum L.) using AFLP markers. Gene 549:171-

178. https://doi.org/10.1016/j.gene.2014.07.067

Chandrawati, Yadav HK (2017) Development of linkage map and mapping of QTLs for oil content and yield attributes in linseed (Linum usitatissimum L.). Euphytica 213:258. https://doi.org/10.1007/s10681-017-2050-x

Cloutier S, Miranda E, Ward K, Radovanovic N, Reimer E, Walichnowski A, Datla R, Rowland G, Duguid S, Ragupathy R (2012) Simple sequence repeat marker development from bacterial artificial chromosome end sequences and expressed sequence tags of flax (Linum usitatissimum L.). Theor Appl Genet 125:685-694. https://doi.org/10.1007/s00122-012-1860-4 
Cloutier S, Niu Z, Datla R, Duguid S (2009) Development and analysis of EST-SSRs for flax (Linum usitatissimum L.). Theor Appl Genet 119:53-63. https://doi.org/10.1007/s00122-009-1016-3

Cloutier S, Ragupathy R, Niu Z, Duguid S (2011) SSR-based linkage map of flax (Linum usitatissimum L.) and mapping of QTLs underlying fatty acid composition traits. Mol Breeding 28:437-451.

https://doi.org/10.1007/s11032-010-9494-1

Earl DA, Vonholdt BM (2012) STRUCTURE HARVESTER: a website and program for visualizing STRUCTURE output and implementing the Evanno method. Conservation Genet Resour 4:359-

361. https:// doi.org/10.1007/s12686-011-9548-7

Eastmond PJ, Quettier AL, Kroon JT, Craddock C, Adams N, Slabas AR (2010) Phosphatidic acid phosphohydrolase 1 and 2 regulate phospholipid synthesis at the endoplasmic reticulum in Arabidopsis. Plant Cell 22:2796-811. https://doi.org/10.1105/tpc.109.071423

Fu Y, Diederichsen A, Richards KW, Peterson G (2002) Genetic diversity within a range of cultivars and landraces of flax (Linum usitatissimum L.) as revealed by RAPD. Genet Resour Crop Evol 49:167-

174. https://doi.org/10.1023/A:1014716031095

Fu Y, Guerin S, Peterson GW, Diederichsen A, Rowland GG, Richards KW (2003) RAPD analysis of genetic variability of regenerated seeds in the canadian flax cultivar CDC normandy. Seed Sci Technol 31:207-211. https://doi.org/10.15258/sst.2003.31.1.22

Guo G, Zhang G, Pan B, Diao W, Liu J, Ge W, Gao C, Zhang Y, Jiang C, Wang S (2019) Development and Application of InDel Markers for Capsicum spp. Based on Whole-Genome Re-Sequencing. Sci Rep 9:3691. https://doi.org/10.1038/s41598-019-40244-y

Hayashi K, Yoshida H, Ashikawa I (2006) Development of PCR-based allele-specific and InDel marker sets for nine rice blast resistance genes. Theor Appl Genet 113:251-260. https://doi.org/10.1007/s00122-006-0290-6

Hoque A, Fiedler JD, Rahman M (2020) Genetic diversity analysis of a flax (Linum usitatissimum L.) global collection. BMC Genomics 21:557. https://doi.org/10.1186/s12864-020-06922-2

Kizil S, Basak M, Guden B, Tosun HS, Uzun B, Yol E (2020) Genome-Wide Discovery of InDel Markers in Sesame (Sesamum indicum L.) Using ddRADSeq. Plants (Basel) 9:1262. https://doi.org/10.3390/plants9101262

Kraakman AT, Niks RE, Van den Berg PM, Stam P, Van Eeuwijk FA (2004) Linkage disequilibrium mapping of yield and yield stability in modern spring barley cultivars. Genetics 168:435-

446. https://doi.org/10.1534/genetics.104.026831

Kumar S, You FM, Cloutier S (2012) Genome wide SNP discovery in flax through next generation sequencing of reduced representation libraries. BMC Genomics 13:684. https://doi.org/10.1186/1471-2164-13-684

Kumari A, Paul S, Sharma V (2017) Genetic diversity analysis using RAPD and ISSR markers revealed discrete genetic makeup in relation to fibre and oil content in Linum usitatissimum L. genotypes. Nucleus 61:45-53.

https://doi.org/10.1007/s13237-017-0206-7

Page 11/19 
Liu B, Wang Y, Zhai W, Deng J, Wang H, Cui Y, Cheng F, Wang X, Wu J (2013) Development of InDel markers for Brassica rapa based on whole-genome re-sequencing. Theor Appl Genet 126:231-239.

https://doi.org/10.1007/s00122-012-1976-6

Liu G, Xu J, Zhang M, Li P, Yang X (2016) Exploiting Illumina sequencing for the development of InDel markers in watermelon (citrullus lanatus). J Hortic Sci Biotech 91: 220-226.

https://doi.org/10.1080/14620316.2015.1133609

Liu K, Muse SV (2005) PowerMarker: an integrated analysis environment for genetic marker analysis. Bioinformatics 21:2128-2129. https://doi.org/10.1093/bioinformatics/bti282

Liu S, An Y, Tong W, Qin X, Samarina L, Guo R, Xia X, Wei C (2019) Characterization of genome-wide genetic variations between two varieties of tea plant (Camellia sinensis) and development of InDel markers for genetic research. BMC Genomics 20:935. https://doi.org/10.1186/s12864-019-6347-0

Lv H, Yang L, Kang J, Wang Q, Wang X, Fang Z, Liu Y, Zhuang M, Zhang Y, Lin Y, Yang Y, Xie B, Liu B, Liu J (2013) Development of InDel markers linked to Fusarium wilt resistance in cabbage. Mol Breeding 32:961-967. https://doi.org/10.1007/s11032-013-9925-x

Mietkiewska E, Siloto RM, Dewald J, Shah S, Brindley DN, Weselake RJ (2011) Lipins from plants are phosphatidate phosphatases that restore lipid synthesis in a pah1 $\Delta$ mutant strain of Saccharomyces cerevisiae. FEBS J 278:764-775. https://doi.org/10.1111/j.1742-4658.2010.07995.x

Murray MG, Thompson WF (1980) Rapid isolation of high molecular weight plant DNA. Nucleic Acids Res 8:4321-4325. https://doi.org/10.1093/nar/8.19.4321

Nakamura Y, Koizumi R, Shui G, Shimojima M, Wenk MR, Ito T, Ohta H (2009) Arabidopsis lipins mediate eukaryotic pathway of lipid metabolism and cope critically with phosphate starvation. Proc Natl Acad Sci U S A 106:20978-20983. https://doi.org/10.1073/pnas.0907173106

Ngan TP, Kim MK, Sim SC (2016) Genetic variations of F1 tomato cultivars revealed by a core set of SSR and InDel markers. Sci Hortic 212:155-161. https://doi.org/10.1016/j.scienta.2016.09.043

Pan G, Chen A, Li J, Huang S, Tang H, Chang L, Zhao L, Li D (2020) Genome-wide development of simple sequence repeats database for flax (Linum usitatissimum L.) and its use for genetic diversity assessment. Genet Resour Crop Evol 67:865-874. https://doi.org/10.1007/s10722-020-00882-y

Sahu PK, Mondal S, Sharma D, Vishwakarma G, Kumar V, Das BK (2017) InDel marker based genetic differentiation and genetic diversity in traditional rice (Oryza sativa L.) landraces of Chhattisgarh, India. PLoS One 12: e0188864. https://doi.org/10.1371/journal.pone.0188864

Singh N, Agarwal N, Yadav HK (2019) Genome-wide SNP-based diversity analysis and association mapping in linseed (Linum usitatissimum L.). Euphytica 215. https://doi.org/10.1007/s10681-019-2462-x

Soto-Cerda BJ, Carrasco RA, Aravena GA, Urbina HA, Navarro CS (2011) Identifying novel polymorphic microsatellites from cultivated flax (Linum usitatissimum L.) following data mining. Plant Mol Biol Report 
Soto-Cerda BJ, Cloutier S, Quian R, Gajardo HA, Olivos M, You FM (2018) Genome-Wide Association Analysis of Mucilage and Hull Content in Flax (Linum usitatissimum L.) Seeds. Int J Mol Sci 19:2870. https://doi.org/10.3390/ijms19102870

Spielmeyer W, Green AG, Bittisnich D, Mendham N, Lagudah ES (1998) Identification of quantitative trait loci contributing to fusarium wilt resistance on an AFLP linkage map of flax (Linum usitatissimum). Theor App Genet 97:633-641. https://doi.org/10.1007/s001220050939

Wang X, Shen F, Gao Y, Wang K, Chen R, Luo J, Yang L, Zhang X, Qiu C, Li W, Wu T, Xu X, Wang Y, Cong P, Han Z, Zhang X (2020) Application of genome-wide insertion/deletion markers on genetic structure analysis and identity signature of Malus accessions. BMC Plant Biol 20:540. https://doi.org/10.1186/s12870-020-02744-2

Wei L, Miao H, Li C, Duan Y, Niu J, Zhang T, Zhao Q, Zhang H (2014) Development of SNP and InDel markers via de novo transcriptome assembly in Sesamum indicum L. Mol Breeding 34(4):2205-2217.

https://doi.org/10.1007/s11032-014-0174-4

Westcott ND, Muir AD (2003) Flax seed lignan in disease prevention and health promotion. Phytochemistry Reviews 2:401-417. https://doi.org/10.1023/B:PHYT.0000046174.97809.b6

Wu D, Wu H, Wang C, Tseng H, Hwu K $\nabla 2013 \otimes$ Genome-wide InDel marker system for application in rice breeding and mapping studies. Euphytica 192ه131-143. https://doi.org/10.1007/s10681-013-0925-Z

Wu J, Zhao Q, Wu G, Zhang S, Jiang T (2017) Development of Novel SSR Markers for Flax (Linum usitatissimum L.) Using Reduced-Representation Genome Sequencing. Front Plant Sci 7:2018. https://doi.org/10.3389/fpls.2016.02018

Wu J, Zhao Q, Zhang L, Li S, Ma Y, Pan L, Lin H, Wu G, Yuan H, Yu Y, Wang X, Yang X, Li Z, Jiang T, Sun D (2018) QTL Mapping of Fiber-Related Traits Based on a High-Density Genetic Map in Flax (Linum usitatissimum L.). Front Plant Sci 9:885. https://doi.org/10.3389/fpls.2018.00885

Xie D, Dai Z, Yang Z, Sun J, Zhao D, Yang X, Zhang L, Tang Q, Su J (2018) Genome-Wide Association Study Identifying Candidate Genes Influencing Important Agronomic Traits of Flax (Linum usitatissimum L.) Using SLAF-seq. Front Plant Sci 8:2232. https://doi.org/10.3389/fpls.2017.02232

Xie D, Dai Z, Yang Z, Tang Q, Deng C, Xu Y, Wang J, Chen J, Zhao D, Zhang S, Zhang S, Su J (2019) Combined genome-wide association analysis and transcriptome sequencing to identify candidate genes for flax seed fatty acid metabolism. Plant Sci 286:98-107. https://doi.org/10.1016/j.plantsci.2019.06.004

Yang Z, Dai Z, Xie D, Chen J, Tang Q, Cheng C, Xu Y, Wang T, Su J (2018) Development of an InDel polymorphism database for jute via comparative transcriptome analysis. Genome 61:323-327. https://doi.org/10.1139/gen-2017-0191

Yi L, Gao F, Siqin B, Zhou Y, Li Q, Zhao X, Jia X, Zhang H (2017) Construction of an SNP-based high-density linkage map for flax (Linum usitatissimum L.) using specific length amplified fragment sequencing (SLAF-seq) 
technology. PLoS One 12: e0189785. https://doi.org/10.1371/journal.pone.0189785

You FM, Xiao J, Li P, Yao Z, Jia G, He L, Kumar S, Soto-Cerda B, Duguid SD, Booker HM, Rashid KY, Cloutier S (2018) Genome-Wide Association Study and Selection Signatures Detect Genomic Regions Associated with Seed Yield and Oil Quality in Flax. Int J Mol Sci 19:2303. https://doi.org/10.3390/ijms19082303

Zhang J, Long Y, Wang L, Dang Z, Zhang T, Song X, Dang Z, Pei X (2018) Consensus genetic linkage map construction and QTL mapping for plant height-related traits in linseed flax (Linum usitatissimum L.). BMC Plant Biol 1:160. https://doi.org/10.1186/s12870-018-1366-6

Zhang M, Yu Q, Liu Z, Liang C, Zhang B, Li M (2017) UBX domain-containing proteins are involved in lipid homeostasis and stress responses in Pichia pastoris. Int J Biochem Cell Biol 90:136-

144. https://doi.org/10.1016/j.biocel.2017.08.006

Zhang T, Gu M, Liu Y, Lv Y, Zhou L, Lu H, Liang S, Bao H, Zhao H (2017) Development of novel InDel markers and genetic diversity in Chenopodium quinoa through whole-genome re-sequencing. BMC Genomics 18:685. https://doi.org/10.1186/s12864-017-4093-8

Zuk M, Richter D, Matuła J, Szopa J (2015) Linseed, the multipurpose plant. Ind Crop Prod 75:165-177. https://doi.org/10.1016/j.indcrop.2015.05.005

\section{Figures}

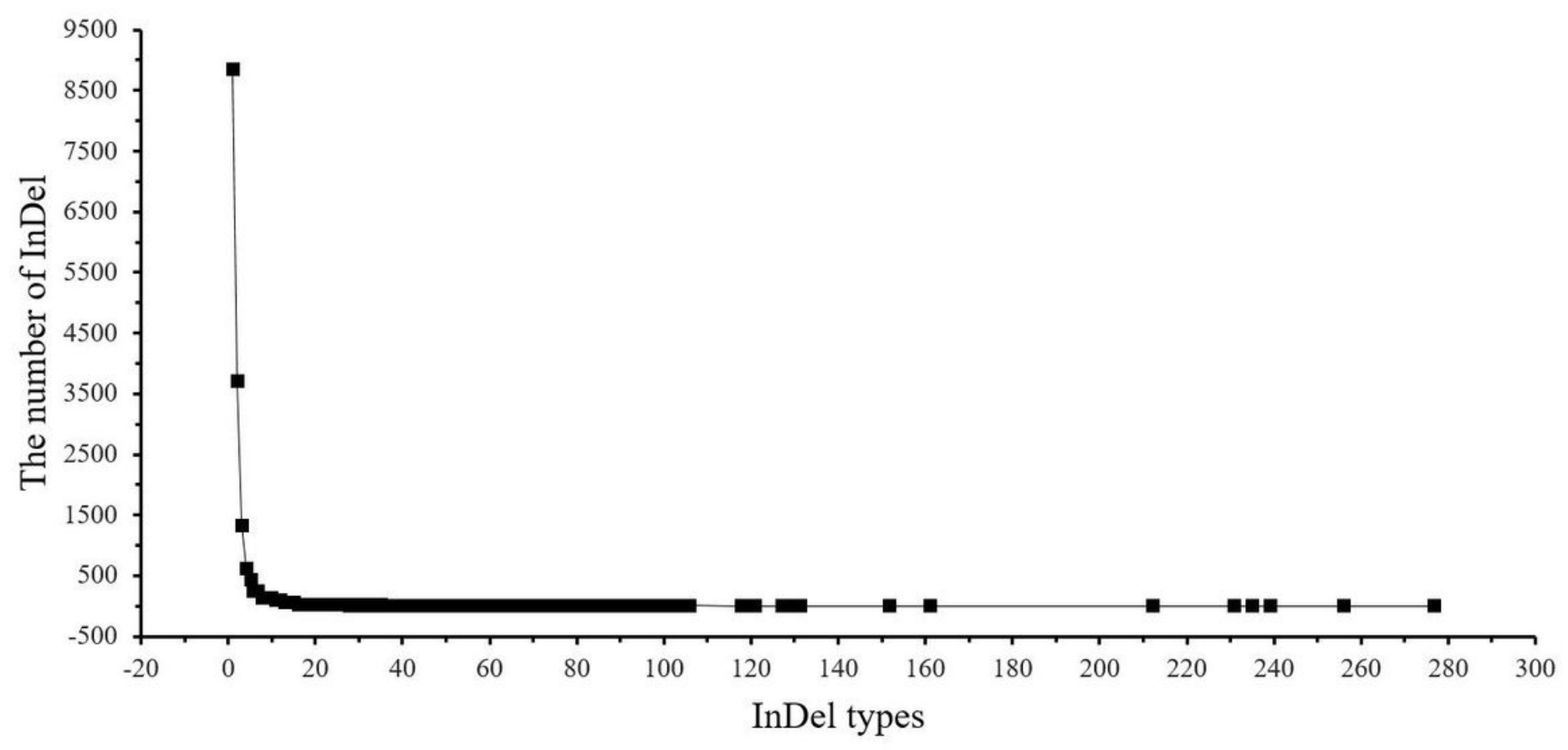

Figure 1

Frequency of InDel type of flax 


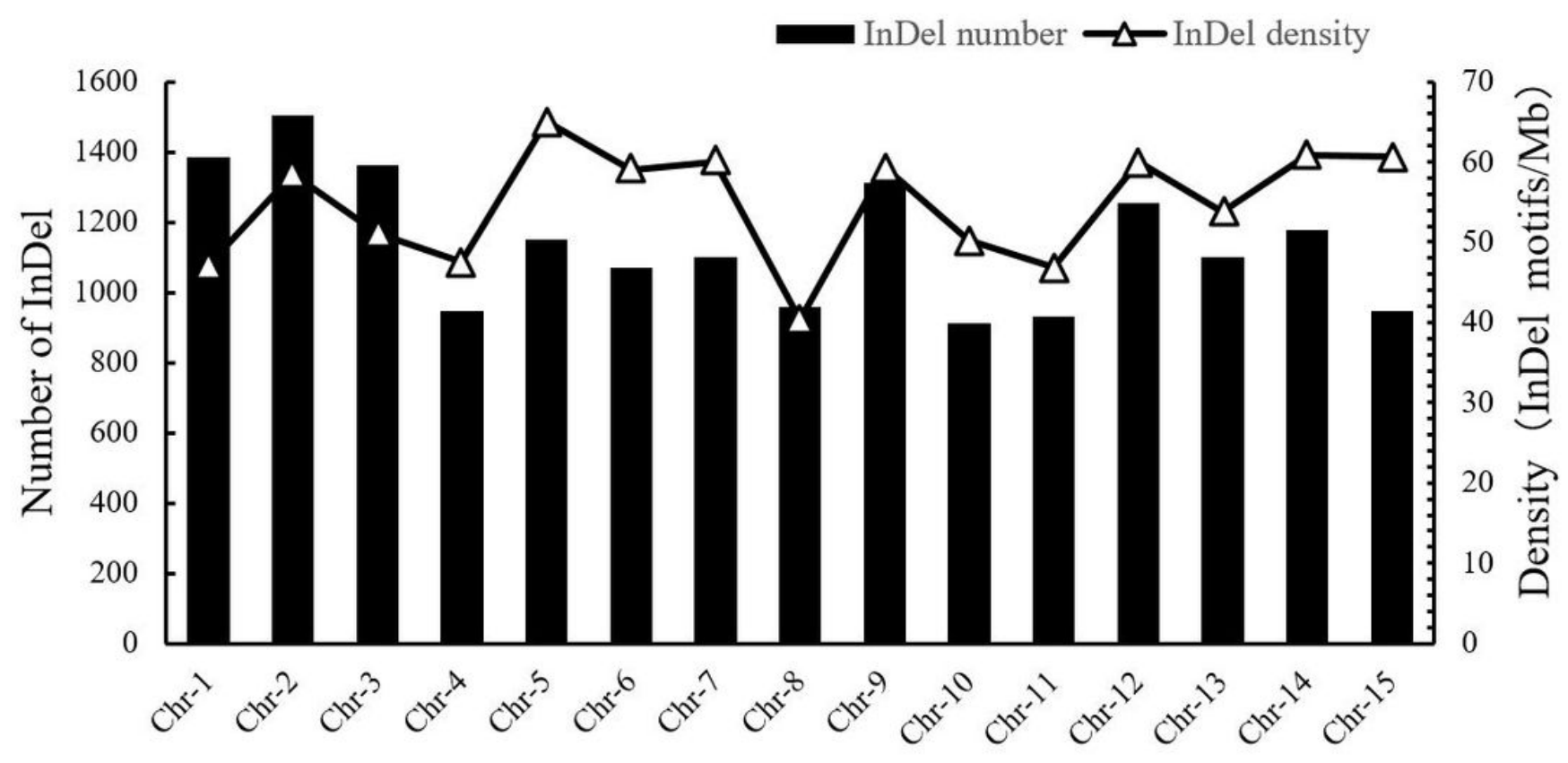

Figure 2

The number of InDel (A) and density of InDel (B) on each chromosome in the flax genome

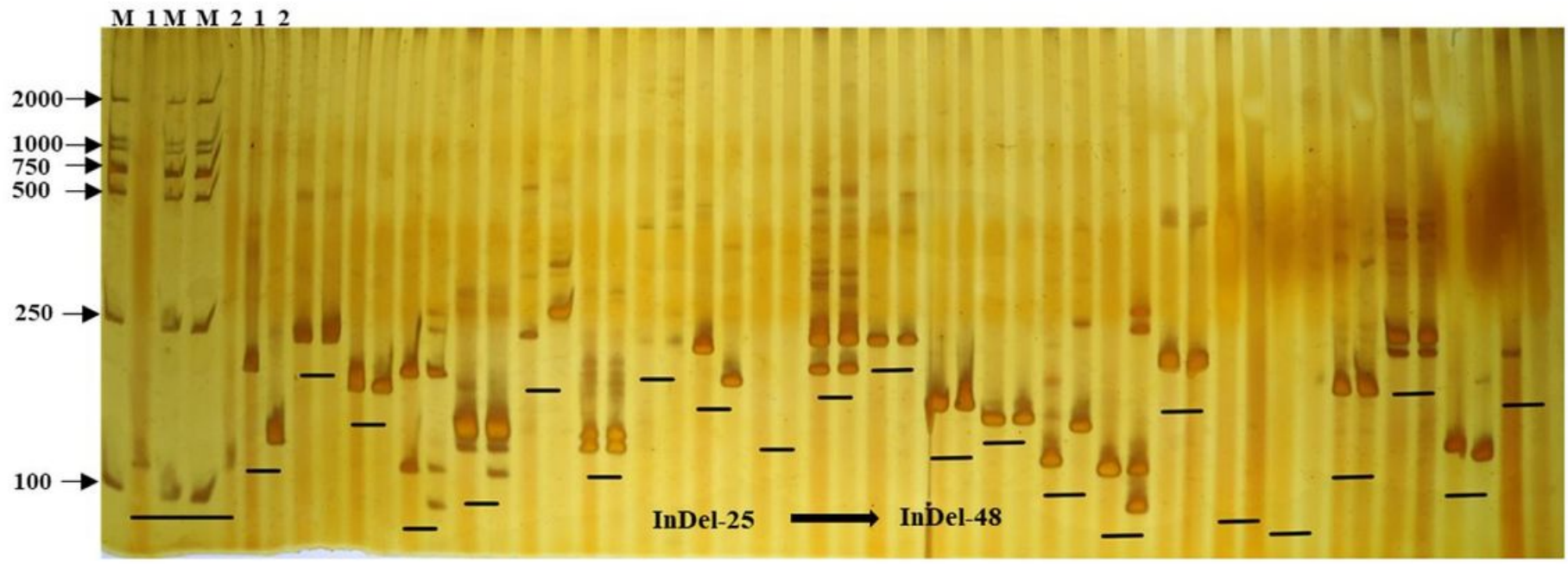

Figure 3

Amplification products in 87-3 and 84-3 with InDel-25-InDel-48. M: 2000bp marker; 1: 87-3, 2: 84-3 


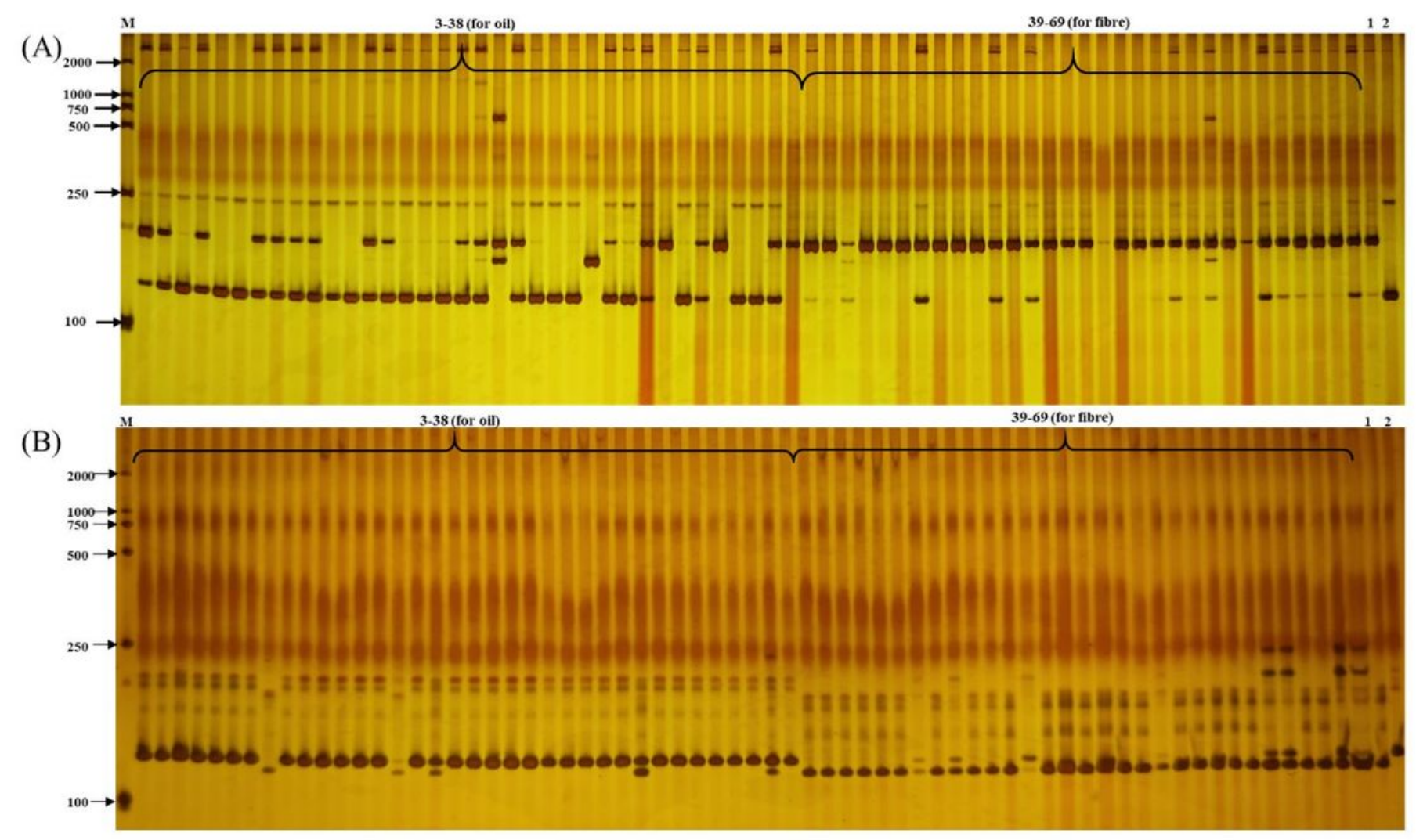

\section{Figure 4}

Amplification products of 69 flax accessions using InDel-26 (A) and InDel-81 (B). a: InDel-26 amplified band; b: InDel-81 amplified band. M: 2000bp marker; 1 and 2 represent male and female respectively; 3-38: flax accessions for oil; 39-69, flax accessions for fibre. 


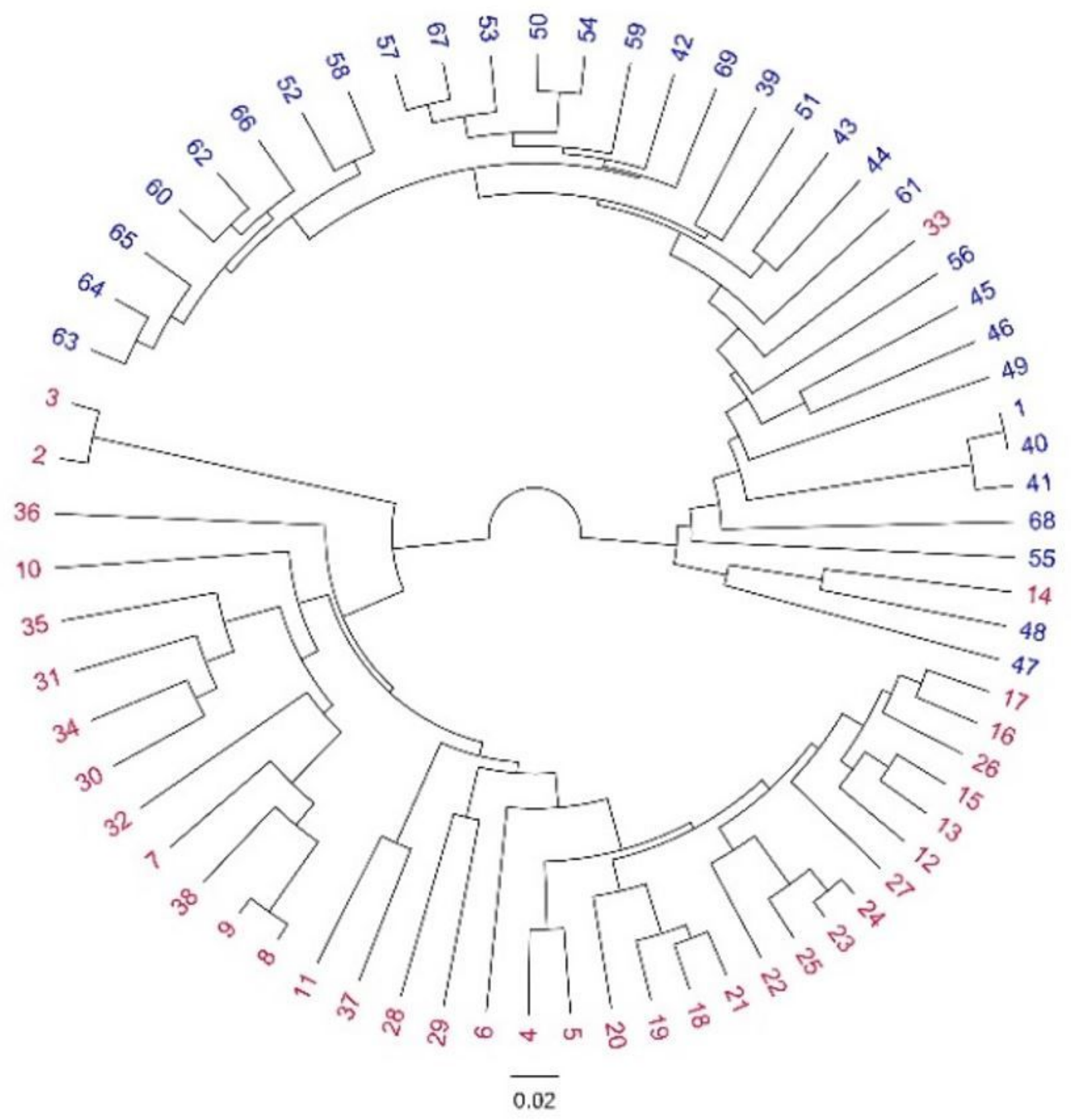

Figure 5

Cluster analyse of 69 flax accessions based on 32 InDel markers; red numbers represent oil flax, blue represent fibre flax 

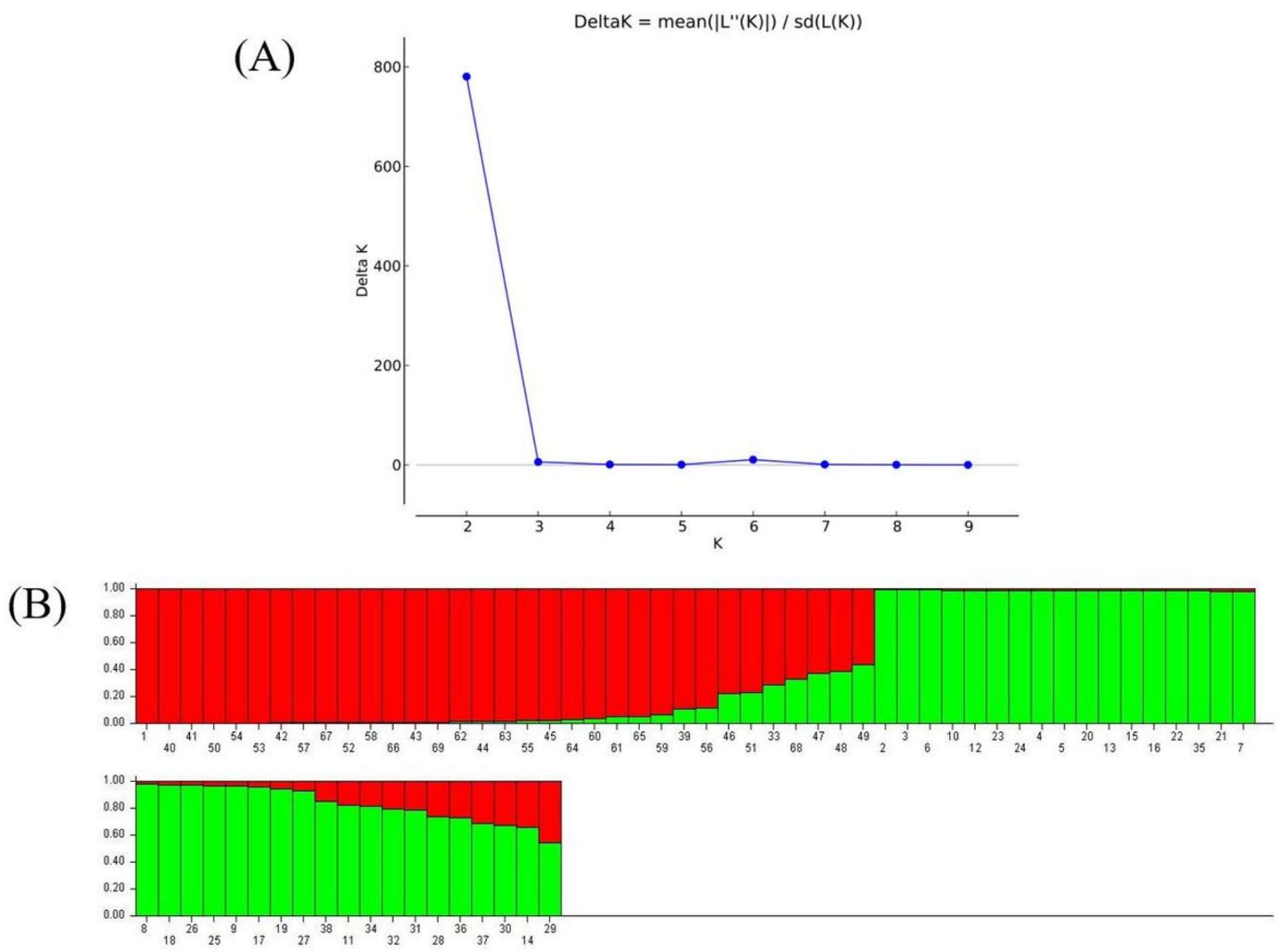

Figure 6

(A) Delta $K$ values for different assumed (K) populations and (B) population structure analyse divided 69 flax accessions into two populations based on 32 InDel markers $(K=2)$ 


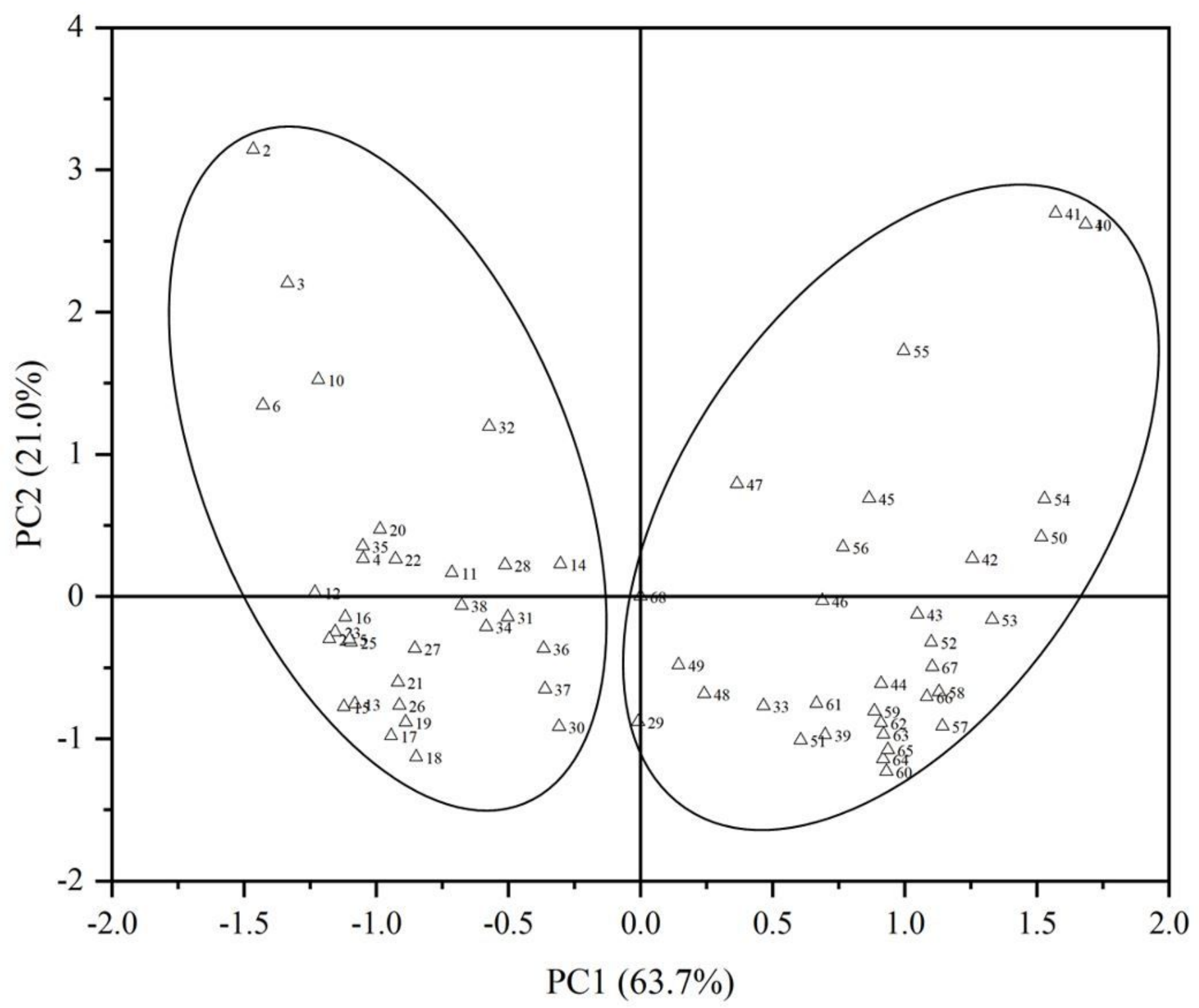

Figure 7

Principal component analysis plot of 69 flax accessions based on 32 InDel markers

\section{Supplementary Files}

This is a list of supplementary files associated with this preprint. Click to download.

- Supplementarydata.docx 